\title{
Application of positron emission tomography-computed tomography in the diagnosis of pulmonary ground-glass nodules
}

\author{
LILI HU, YUANWEI PAN, ZHIGANG ZHOU and JIANBO GAO
}

Department of Radiology, The First Affiliated Hospital of Zhengzhou University, Zhengzhou, Henan 450052, P.R. China

Received September 29, 2016; Accepted June 15, 2017

DOI: $10.3892 / e t m .2017 .5194$

\begin{abstract}
The aim of the present study was to investigate the value of positron emission tomography-computed tomography (PET-CT) using ${ }^{18} \mathrm{~F}$-fluorodeoxyglucose in the clinical diagnosis of pulmonary ground-glass nodule. In total, 54 patients with pulmonary GGN that were identified by PET-CT examination were selected and confirmed by pathology and clinical diagnosis in hospital between April 2014 and April 2015. The association between PET-CT findings and pathology, and the value of PET-CT were then evaluated. In the 54 patients, solitary pulmonary GGN with a nodule diameter of between 0.6 and $2.0 \mathrm{~cm}$ were detected. Amongst them, the PET-CT examination of 42 patients revealed hyper metabolic nodules, and were all mixed GGN type nodules with a diameter $>1 \mathrm{~cm}$. The PET-CT examination of the remaining 12 patients demonstrated no evidence of metabolic abnormalities and the nodules in these patients were pure or mixed GGN with a diameter $<1 \mathrm{~cm}$ (except 2 cases with a diameter $\geq 1 \mathrm{~cm}$ ). Furthermore, the diagnoses for all patients were pathologically confirmed by CT-guided needle biopsy or thoracoscopic surgical resection. Amongst them, there were 41 cases of lung adenocarcinoma, 4 cases of fungal infection, 7 cases of inflammation and 2 cases of adenomatoid hyperplasia. Additionally, PET-CT has a lower detection rate for smaller GGN exhibits no clear advantage for pure GGN, but has a higher detection rate for larger GGN. In conclusion, to a certain extent, PET-CT makes up for the shortcomings of traditional imaging and has some clinical value for the diagnosis of GGN.
\end{abstract}

\section{Introduction}

With the development of computed tomography (CT) and positron emission tomography (PET), the number of patients

Correspondence to: Dr Jianbo Gao, Department of Radiology, The First Affiliated Hospital of Zhengzhou University, 1 Jianshe East Road, Zhengzhou, Henan 450052, P.R. China

E-mail: gaojianbo2016@126.com

Key words: pulmonary ground-glass nodule, positron emission tomography-computed tomography, ${ }^{18} \mathrm{~F}$-fluorodeoxyglucose, tomography, X-ray computed tomography who are characterized by ground-glass nodule (GGN) of CT has increased (1). GGN is a pulmonary nodule characterized by ground-glass opacity, which refers to high-density shadows on CT images and visible pulmonary bronchial or pulmonary vessels of the lung (2). Numerous pulmonary diseases are characterized by GGN on CT, such as early lung cancer, pneumonia, alveolar hemorrhage (3). However, the differential diagnosis of benign and malignant lesions is currently a focus of research due to the complexity of the imaging of pulmonary GGN (4). GGN occurs most frequently in bronchioloalveolar carcinoma, adenocarcinoma and atypical adenomatous hyperplasia. However, it also occurs in inflammatory and focal lesions, focal hemorrhage or fibrosis (4). Therefore, pulmonary GGN are still difficult to identify.

PET-CT is able to observe the metabolic changes of diseases from the molecular level, and has broad application prospects in cancer (5). PET-CT could analyze the distribution range, appearance, shape and companion of GGN. It could also identify accompanying signs and dynamic characteristics (6). Therefore, it will help to narrow the scope of diagnosis and provide clues for the diagnosis. At present, it is widely used in the diagnosis, staging, therapeutic evaluation and recurrence monitoring of cancer (7).

In the present study, the PET-CT examination results of 54 patients with pulmonary GGN were studied and compared with the pathological observations with the purpose of evaluating the application value of PET-CT in the diagnosis of pulmonary GGN.

\section{Materials and methods}

General information. In total, 54 patients with pulmonary GGN that were identified by a PET-CT examination were selected and confirmed by pathological examination and clinical diagnosis in the First Affiliated Hospital of Zhengzhou University (Zhengzhou, China) between April 2014 and April 2015. The patients included 32 males and 22 females, aged 32-68 years. Inclusion criteria for patients were as follows: i) Pulmonary nodules identified by CT; ii) no previous history of cancer; and iii) blood sugar levels were maintained at normal levels. Exclusion criteria were as follows: i) Diabetic patients whose glucose could not be controlled in normal range; and ii) pregnant or lactating females.

All diagnoses were confirmed by the examination of pathological slices, which were acquired through CT-guided 
needle biopsy or thoracoscopic local excision of the lesion. All patients were required to fast for $4-6 \mathrm{~h}$ prior to the examination. Blood sugar, height and body mass were detected before examination. The patients were requested to be in repose for 40-60 min after intravenous injection. Then, the clavicle, axillary and double pulmonary fields were scanned by spiral CT. If the image of the internal and edge of GGN was poor, target scan or high resolution CT scan was performed. The present study was ethically approved by the Ethics Committee of The First Affiliated Hospital of Zhengzhou, and written informed consent was obtained from the patients.

Examination methods. A Siemens Biograph Truepoint 64 (52 rings) PET-CT instrument (Siemens AG, Munich, Germany) with a 52-ring lutetium oxyorthosilicate crystal detector and with 32,448 detection units was used. The axial field-of-view was $216 \mathrm{~mm}$ and the axial resolution was $2 \mathrm{~mm}$. The integrated CT system had a 64-layer data acquisition system and an instantaneous time resolution of $83 \mathrm{msec}$. The imaging agent used was ${ }^{18} \mathrm{~F}$-fluorodeoxyglucose $\left({ }^{18} \mathrm{~F}-\mathrm{FDG}\right)$, which was produced by Beijing Science and Technology Co., Ltd. (Beijing, China) using a Sumitomo HM-20 medical cyclotron with PET-FDG IT-1 automatic synthesis modules (Sumitomo Heavy Industries. Ltd., Tokyo, Japan). The radioactive chemical purity was $>95 \%$. All patients were required to fast for $4-6 \mathrm{~h}$ prior to the examination and the fasting blood glucose was required to be kept within the normal range (blood sugar concentration was controlled at $7.0 \mathrm{mmol} / \mathrm{l})$. Following the intravenous administration of the ${ }^{18} \mathrm{~F}-\mathrm{FDG}$ imaging agent at a dose of $0.12 \mathrm{mCi} / \mathrm{kg}(8)$, the patient rested in a quiet and dark environment for $60 \mathrm{~min}$, then emptied his/her bladder and drank $800-1,000 \mathrm{ml}$ warm water prior to the scan.

Following confirmation that the PET and CT axial images matched each other through a positioning scan, the patients underwent a whole-body CT scan with the following conditions: $120 \mathrm{kV}$ voltage, 50-80 mA current and $3 \mathrm{~mm}$ layer thickness. The patients then underwent a PET scan. Finally, a processing workstation with TrueD software (Xeleris Workstation v3.0; GE Healthcare, Chicago, IL, USA) was used to conduct image fusion and obtain the axial, coronal and sagittal views for PET, $\mathrm{CT}$ and image fusion.

Image evaluation. A total of three radiology doctors who had $>6$ years of experience in the imaging diagnosis of chest diseases judged the position, size, shape, edge, interface and standardized uptake value of pulmonary lesions and divided these into pure and mixed type GGN according to whether the nodule excluded or contained solid components, respectively. The uptake of ${ }^{18} \mathrm{~F}-\mathrm{FDG}$ in the nodules was observed and compared with the uptake of the pulmonary background. An uptake that was higher than that of the pulmonary background was evaluated as a malignancy, while an uptake lower than or equal to the pulmonary background was evaluated as benign. This result, combined with the imaging findings of GGN lesions, was used to make a diagnosis.

Data analysis. Data were analyzed using t-test and $\chi^{2}$ tests on SPSS statistical analysis software, version 17.0 (SPSS, Inc., Chicago, IL, USA). Data were given as
Table I. Association of the size and type of GGN with benign and malignant lesions according to their metabolism determined using positron emission tomography-computed tomography.

\begin{tabular}{lccccc}
\hline & \multicolumn{2}{c}{$\begin{array}{c}\text { Elevated } \\
\text { metabolism (n) }\end{array}$} & & \multicolumn{2}{c}{$\begin{array}{c}\text { Normal } \\
\text { metabolism (n) }\end{array}$} \\
\cline { 2 - 3 } \cline { 5 - 6 } GGN properties & Benign & Malignant & & Benign & Malignant \\
\hline $\begin{array}{l}\text { Size }(\mathrm{cm}) \\
\geq 1\end{array}$ & 8 & 34 & & 0 & 2 \\
$<1$ & 0 & 0 & & 5 & 5 \\
$\begin{array}{l}\text { Type } \\
\begin{array}{l}\text { Pure ground- } \\
\text { glass type }\end{array}\end{array}$ & 0 & 0 & & 2 & 4 \\
Mixed type & 8 & 34 & & 3 & 3 \\
\hline
\end{tabular}

GGN, ground-glass nodule.

mean \pm standard deviation. All the results were repeated three times. $\mathrm{P}<0.05$ was considered to indicate a statistically significant result.

\section{Results}

General information. The 54 patients included 32 male and 22 female cases and the age range of the patients was 32-68 years. All patients had solitary pulmonary GGN, which were distributed in the upper lobe of the left lung in 12 cases, in the lower lobe of the left lung in 8 cases, in the upper lobe of the right lung in 24 cases, in the middle lobe of the right lung in 3 cases and in the lower lobe of the right lung in 7 cases. The nodule size range was $0.6-2.0 \mathrm{~cm}$.

The 54 cases of GGN included 44 cases where the GGN had a diameter $>1 \mathrm{~cm}$. Among these, 2 cases were pure GGN without elevated radioactivity uptake and the remaining 42 cases were mixed type GGN with an elevated metabolism compared with the pulmonary background. There were 10 cases of GGN with a diameter of $<1 \mathrm{~cm}$, including 5 cases of mixed nodules and 5 cases of pure GGN, all of which did not have elevated radioactive uptake (Table I).

Association between pathological type and metabolic observations. The association between pathological type and nodule metabolism is demonstrated in Table II. Positive rate refers to the PET-CT diagnosis of malignant lesions as a proportion of malignant cases; detection rate refers to the PET-CT diagnosis of benign lesions as a proportion of cases; false positive rate refers to the PET-CT diagnosis of benign cases as a proportion of malignant cases. By analysis of the data in this table, the detection rate of PET-CT for malignant lesions in the study population can thus be calculated to be $62.9 \%$, with a positive rate of $82.9 \%$ and a false positive rate of $58.3 \%$.

Associations of the size and type of GGN with metabolic observations and pathology. The associations of the size of the GGN with the metabolic observations provided by PET-CT and the 

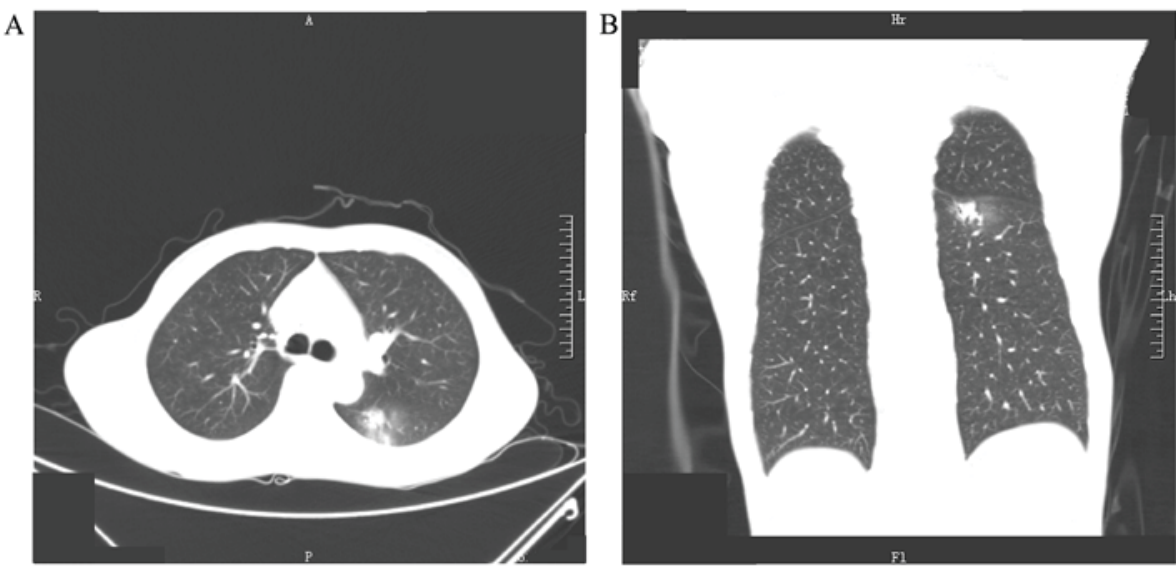

Figure 1. Computed tomography images of GGN in the left lung at admission. (A) GGN in the lower lobe of the left lung, observed from axial view. (B) GGN in the lower lobe of the left lung, observed from coronal view. The postoperative pathology was adenocarcinoma. GGN, ground-glass nodule.

Table II. Association between the pathological type of ground-glass nodule and PET-CT results.

\begin{tabular}{lcc}
\hline & \multicolumn{2}{c}{ PET-CT (n) } \\
\cline { 2 - 3 } $\begin{array}{l}\text { Pathological } \\
\text { type }\end{array}$ & $\begin{array}{c}\text { Elevated } \\
\text { metabolism }\end{array}$ & $\begin{array}{c}\text { Normal } \\
\text { metabolism }\end{array}$ \\
\hline $\begin{array}{l}\text { Adenocarcinoma } \\
\text { Atypical adenomatous } \\
\text { hyperplasia }\end{array}$ & 34 & 7 \\
Inflammation & 2 & 0 \\
Fungal infection & 5 & 2 \\
\hline
\end{tabular}

PET-CT, positron emission tomography-computed tomography.

Table III. Association between imaging observations and the benign and malignant nature of the nodules.

\begin{tabular}{lcccc}
\hline Observations & Malignant & Benign & $\chi^{2}$-test & P-value \\
\hline Size $(\mathrm{cm})$ & $1.2 \pm 0.8$ & $1.0 \pm 0.4$ & & \\
Regular shape & 27 & 16 & 5.993 & 0.014 \\
Burr-like & 32 & 1 & 20.559 & $<0.01$ \\
Lobulated & 24 & 3 & 4.964 & 0.026 \\
Vascular bundle & 34 & 8 & 2.612 & 0.106 \\
Pleural indentation & 26 & 2 & 9.120 & 0.003 \\
Smooth edges & 7 & 23 & 4.567 & 0.033 \\
Vacuole sign & 22 & 2 & 5.856 & 0.016 \\
\hline
\end{tabular}

pathology results were analyzed and are presented in Table I. Amongst the lesions with a diameter $>1 \mathrm{~cm}$, the PET-CT of 42 cases indicated a high metabolism, of which $81 \%(34 / 42)$ were malignant and 19\% (8/42) were benign. Additionally, the PET-CT scans of 2 malignant cases did not reveal elevated metabolism. There were 10 lesions with a diameter $<1 \mathrm{~cm}$, of which 50\% (5/10) were malignant and 50\% (5/10) were benign. None of these 10 lesions exhibited elevated metabolism.
Results analyzed according to the type of GGN are also presented in Table I. There were 7 cases of pure GGN without elevated metabolism, among which 2 cases were benign and 5 cases were malignant. The remaining 47 cases were mixed nodules, and 42 of these cases had a high PET-CT metabolism, 8 of which were benign and 34 of which were malignant. Among the 5 cases of mixed GGN without elevated metabolism, 3 cases were benign and 2 cases were malignant.

Association of imaging observations with nodule malignancy. The associations between imaging observations and the benign and malignant features of local nodules are demonstrated in Table III. From these results, it may be observed that the mean size of the malignant GGN was greater than that of those that were benign. Furthermore, nodules with a regular shape were more likely to be malignant while those with a smooth edge were more likely to be benign. By further analyzing the imaging features of the GGN, the presence of a vacuole sign, pleural indentation signs, lobulation and burr-like appearance indicated a higher possibility of the lesion being malignant rather than benign. Examples of imaging scans for various patients are shown in Figs. 1-4.

\section{Discussion}

With the popularization and application of high-end CT scanning and the enhancement of people's health consciousness, the detection rate of pulmonary nodules is increasing (9). A multinomial study reported that the sensitivity and specificity of the diagnosis of primary lung cancer by PET-CT were $84-97$ and $76-95 \%$, respectively (10). However, the diagnosis of GGN is challenging due to the poor specificity of their imaging performance. In 1997, the Nomenclature Committee of the International Union of Biochemistry defined GGN as a fuzzy density where the bronchi or lung blood vessels remain visible with high-resolution CT (11). Additionally, it refers to a class of non-specific CT signs that have a slightly increased density in high-resolution CT images (12). With regard to pathology, GGN has been identified for a variety of inflammatory conditions, edema, bleeding, fibrosis and tumor lesions (13-17).

For smaller pulmonary GGN lesions, the National Comprehensive Cancer Network (18) suggest that lesions that 


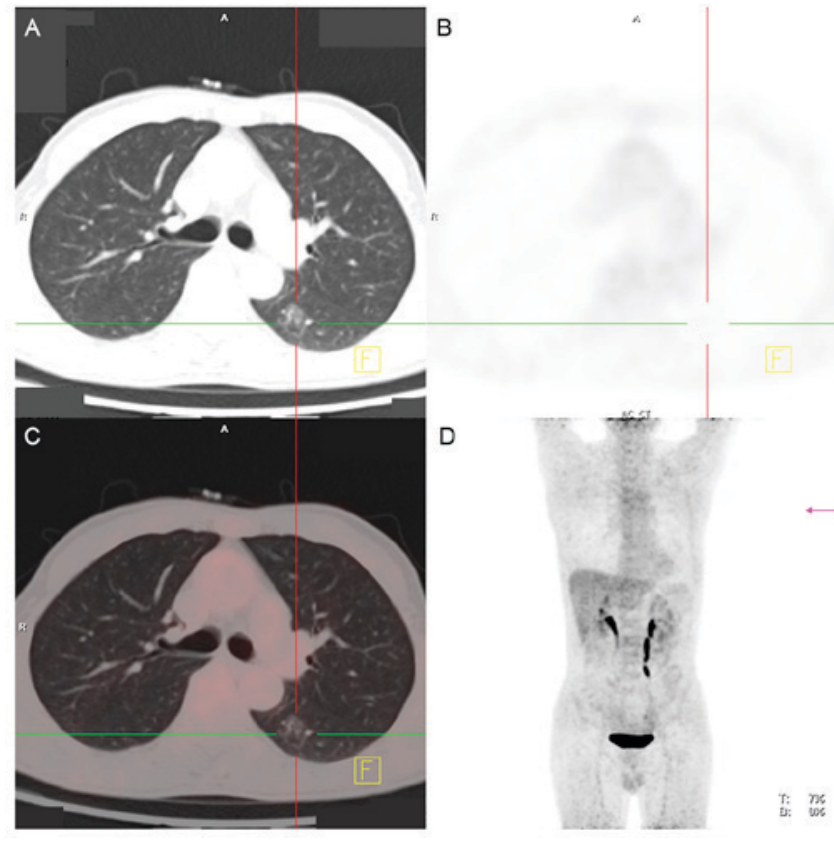

Figure 2. Positron emission tomography-CT images of GGN in the left lung at admission. (A) CT image of GGN in the left lower lobe. (B) Functional metabolic image, indicating that nodules have low metabolism. (C) CT and metabolic fusion image of maximal intensity projection. (D) Functional metabolic image. CT, computed tomography; GGN, ground-glass nodule.

are $>0.8 \mathrm{~cm}$ and persistently exist should be dealt with as soon as possible, while the lesions that are $<0.8 \mathrm{~cm}$ should be dynamically observed and surgically biopsied. Therefore, the judgment of whether the imaging shows benign or malignant GGN lesions directly affects the patient's treatment options. As PET-CT is becoming more widely used, the research on its use in the diagnosis of pulmonary GGN is increasing (1). In vitro and in vivo experiments have demonstrated that lung cancer cells have higher levels of glucose metabolism as compared with normal tissue cells (19). Furthermore, as an effective means of identification and diagnosis, ${ }^{18} \mathrm{~F}$-FDG PET-CT is important in the diagnosis of primary lung cancer (20). Previous studies revealed the sensitivity and specificity for ${ }^{18} \mathrm{~F}-\mathrm{FDG}$ PET (PET-CT) in the diagnosis of lung cancer primary lesions to be $84-97$ and 76-95\%, respectively (21-23). Additionally, the diagnostic value of PET-CT has been affirmed by numerous researchers, particularly for nodular lesions that were otherwise difficult to characterize $(7,24)$.

At present, ${ }^{18} \mathrm{~F}-\mathrm{FDG}$ is the most widely used imaging agent in PET-CT imaging in the clinic. Tumors use glucose mainly in glycolysis to obtain energy, even when adequate oxygen is available. Furthermore, the use of glycolysis as the main method of obtaining energy by tumors is associated with the existence of intermittent hypoxia in tumor tissues (25). Hypoxia induces an increase in the expression of the glucose transporter-1, thereby increasing glucose uptake (26). Tumor hypoxia may be associated with numerous other factors, including abnormal morphology and function of the microvasculature that feeds the tumor, an increased distance between nutrient vessels and tumor cells, reduction of the oxygen transfer capacity of red blood cells induced by the disease itself or therapy, immature tumor cell differentiation and a low capacity of oxygen utilization (27). Tumor cells have high

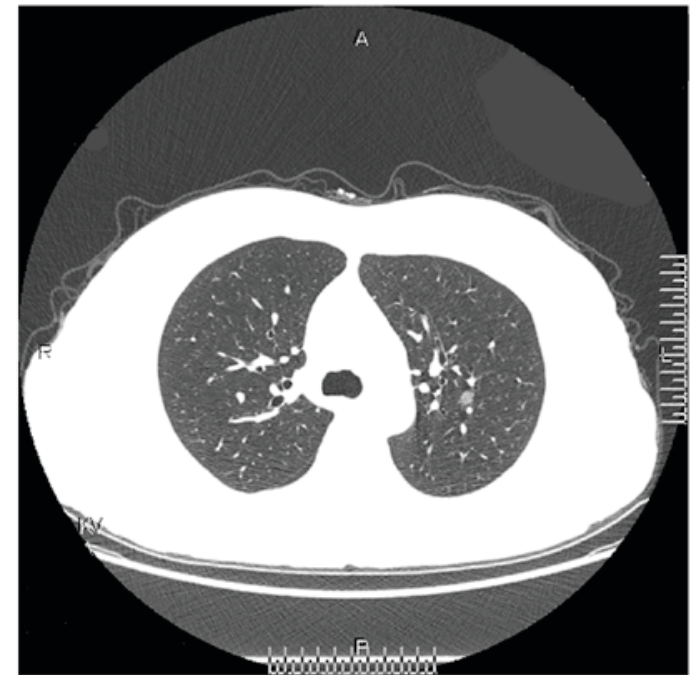

Figure 3. Pure ground-grass nodule was observed on the left upper lobe of the lung. The postoperative pathology confirmed atypical adenomatous hyperplasia.

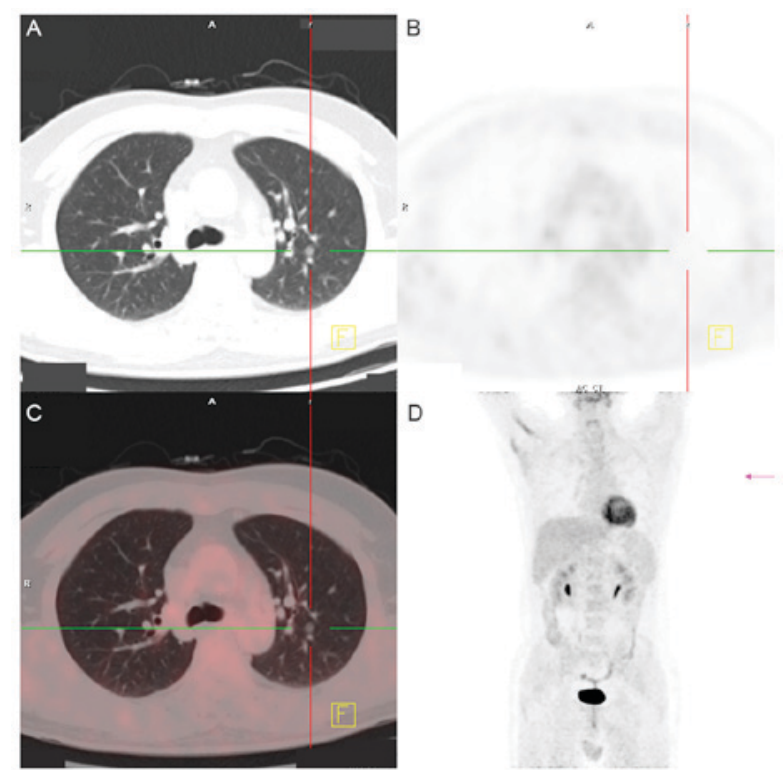

Figure 4. Positron emission tomography-CT images of GGN in the left lung at admission. (A) CT image, the left upper lobe exhibited one GGN (B) Functional metabolic image, indicating that nodules have no metabolism. (C) CT and metabolic fusion image. (D) Functional metabolic image. CT, computed tomography; GGN, ground-glass nodule.

metabolic activity, which causes them to consume a large amount of FDG (28). The ability of cancer cells to undergo glycolysis is associated with the degree of malignancy and the proliferation rate of the tumor; specifically, tumor cells with a high degree of malignancy, strong invasiveness and high proliferation rate have greater glycolytic activity (29).

In the present study, there were 54 GGN lesions, ranging in diameter from 0.6 to $2.0 \mathrm{~cm}$. Among these cases, all 10 lesions with a diameter of $<1 \mathrm{~cm}$ exhibited no evident increase in metabolic uptake, despite them including 5 malignant cases exhibiting an adherent growth type of adenocarcinoma (primary bronchial alveolar carcinoma). Furthermore, they were all considered to be pure GGN, indicating that the lower 
degree of malignancy may be associated with a lower glucose uptake. In the present study, the accuracy of PET-CT was analyzed according to the nodule diameter and the type of nodule. It was concluded that the detection rate of PET-CT was low for smaller GGN, and there was no clear advantage for pure GGN, while the detection rate of PET-CT was high for larger GGN. However, due to the small number of cases with a diameter $<1 \mathrm{~cm}$, expansion of the sample size is necessary for this conclusion to be verified.

In conclusion, PET-CT is an examination method that combines pathological metabolic patterns with imaging manifestations and has been widely used in the examination of malignant diseases. When PET-CT is used to judge the benign and malignant nature of pulmonary GGN, a comprehensive diagnosis should be performed by taking into account the nodule size, imaging performance, glucose metabolism and clinical data. The sample size used in the current study is small, and for smaller diameter, pure GGN, the number of cases examined requires expansion in order to clarify the findings.

\section{Acknowledgements}

The authors would like to express their gratitude to all those who helped during the writing up of the present study.

\section{References}

1. Gao YJ and Zsolt Szabo: Metabolic and anatomic characteristics of bronchioloalveolar carcinoma on 18F-FDG PET/CT. Chin J Nucl Med 29: 238-241, 2009

2. Naidich DP, Bankier AA, MacMahon H, Schaefer-Prokop CM, Pistolesi M, Goo JM, Macchiarini P, Crapo JD, Herold CJ, Austin JH and Travis WD: Recommendations for the management of subsolid pulmonary nodules detected at CT: A statement from the fleishchner society. Radiology 266: 304-317, 2013

3. Kim H, Park CM, Koh JM, Lee SM and Goo JM: Pulmonary subsolid nodules: What radiologists need to know about the imaging features and management strategy. Diagn Interv Radiol 20: 47-57, 2014.

4. Park CM, Goo JM, Lee HJ, Lee CH, Chun EJ and Im JG: Nodular Ground-glass opacity at thin-section CT: Histologic correlation and evaluation of change at follow up. Radiographics 27 391-408, 2007

5. Eglinton T, Luck A, Bartholomeusz D, Varghese R and Lawrence M: Positron-emission tomography/computed tomography $(\mathrm{PET} / \mathrm{CT})$ in the initial staging of primary rectal cancer. Colorectal Dis 12: 667-673, 2010.

6. Jung W, Jang JY, Kang MJ, Chang YR, Shin YC, Chang J and Kim SW: The clinical usefulness of $18 \mathrm{~F}$-fluorodeoxyglucose positron emission tomography-computed tomography (PET-CT) in follow-up of curatively resected pancreatic cancer patients. HPB (Oxford) 18: 57-64, 2016.

7. Mac Manus MP and Hicks RJ: PET scanning in lung cancer: Current status and future directions. Semin Surg Oneol 21: $149-155,2003$

8. Liu W, Li X, Quan J, Ouyang X and Zheng H: Diagnostic value of 18F-FDG PET/CT for solitary nodular-type bronchoalveolar carcinoma. Nan Fang Yi Ke Da Xue Xue Bao 33: 114-116, 2013 (In Chinese).

9. Dalli A, Selimoglu Sen H, Coskunsel M, Komek H, Abakay O, Sergi $\mathrm{C}$ and Cetin Tanrikulu A: Diagnostic value of PET/CT in differentiating benign from malignant solitary pulmonary nodules. J BUON 18: 935-941, 2013.
10. Christensen JA, Nathan MA, Mullan BP, Hartman TE, Swensen SJ and Lowe VJ: Characterization of the solitary pulmonary nodule: 18F FDG PET versus nodule-enhancement CT. AJR Am J Roentgenol 187: 1361-1367, 2006.

11. Collins J and Stern EJ: Ground-glass opacity at CT: The ABCs. AJR Am J Roentgenol 169: 355-367, 1997.

12. Kandemir Z, Sentürk A, Ozdemir E, Yildirim N, Hasanoğlu HC, Keskin $\mathrm{M}$ and Türkölmez S: The evaluation of hypermetabolic mediastinal-hilar lymph nodes determined by PET/CT in pulmonary and extrapulmonary malignancies: Correlation with EBUS-TBNA. Turk J Med Sci 45: 1234-1242, 2015.

13. Park CM, Goo JM, Lee HJ, Lee CH, Chun EJ and Im JG: Nodular ground-glass opacity at thin-section CT: Histologic correlation and evaluation of change at follow-up. Radiographics 27: 391-408, 2007.

14. Lei Z, Xu N, Zhang J, et al: MSCT diagnosis of unusual interstitial pneumonia. Chin J Mod Med 22: 76-79, 2012.

15. Qi YG, Huang Y, Zheng JS, et al: The Features and diagnostic value of CT and PET in pneumonia type of bronchioloalveolar carcinoma. J Clin Radic 29: 187-190, 2010.

16. Guo G, Chen N, Li GF, et al: Complete video-assisted thoracoscopic surgery for the diagnosis and treatment of focal ground-glass opacity. Chin J Min Inv Surg 12: 641-643, 2012

17. Jiang BG and Li L: Characteristics of thin slice spiral CT imaging in 45 cases of solitary pulmonary nodule. Chin J Geront 31 : 1541-1543, 2011

18. Porcu P: A look at the national comprehensive cancer network guidelines for cutaneous lymphomas. Clin Lymphoma Myeloma Leuk 10 (Suppl 2): S109-S111, 2010.

19. Lin CC, Chen LC, Tseng VS, Yan JJ, Lai WW, Su WP, Lin CH, Huang CY and Su WC: Malignant pleural effusion cells show aberrant glucose metabolism gene expression. Eur Respir J 37: 1453-1465, 2011.

20. Erasmus JJ, McAdams HP, Rossi SE, Goodman PC, Coleman RE and Patz EF: FDG PET of pleural effusions in patients with non-small cell lung cancer. AJR Am J Roentgenol 175: 245-249, 2000.

21. Endo K, Oriuchi N, Higuchi T, Iida Y, Hanaoka H, Miyakubo M, Ishikita T and Koyama K: PET and PET/CT using 18 F-FDG in the diagnosis and management of cancer patients. Int J Clin Oncol 11: 286-296, 2006

22. Glaudemans AW, de Vries EF, Galli F, Dierckx RA, Slart RH and Signore A: The use of (18)F-FDG-PET/CT for diagnosis and treatment monitoring of inflammatory and infectious diseases. Clin Dev Immunol 2013: 623036, 2013.

23. Mi B, Wan W, Yu C, You X, Jiang F and You Q: The value of extra-lung lesions on ${ }^{8} \mathrm{~F}-\mathrm{FDG}$ PET/CT in improving diagnosis of lung cancer. Zhongguo Fei Ai Za Zhi 15: 78-83, 2012 (In Chinese).

24. Birim O, Kappetein AP, Stijnen T and Bogers AJ: Meta-analysis of positron emission tomographic and computed tomographic imaging in detecting mediastinal lymph node metastases in nonsmall cell lung cancer. Ann Thorac Surg 79: 375-382, 2005.

25. Waki A, Fujibayashi Y, Yonekura Y, Sadato N, Ishii Y and Yokoyama A: Reassessment of FDG uptake in tumor cells: High FDG uptake as a reflection of oxygen-independent glycolysis dominant energy production. Nucl Med Biol 24: 665-670, 1997.

26. Macheda ML, Rogers S and Best JD: Molecular and cellular regulation of glucose transporter (GLUT) proteins in cancer. J Cell Physiol 202: 654-662,2005.

27. Arabi $M$ and Piert M: Hypoxia PET/CT imaging: Implications for radiation oncology. Q J Nucl Mol Imaging 54: 500-509, 2010.

28. Chan WK, Au WY, Wong CY, Liang R, Leung AY, Kwong YL and Khong PL: Metabolic activity measured by F-18 FDG PET in natural killer-cell lymphoma compared to aggressive B- and T-cell lymphomas. Clin Nucl Med 35: 571-575, 2010.

29. Koiso K, Kakizoe T, Murahashi I, Umeda T and Ueno A: Studies on the metabolic activities of the bladder tumors. 2. Studies on the energy metabolism of the bladder tumors (author's transl). Nihon Hinyokika Gakkai Zasshi 68: 22-32, 1977 (In Japanese). 